\title{
Mammary Mastectomy Due to Mammary Gland Tumors in Intact Female Dog
}

\author{
Haben Fesseha* \\ School of Veterinary Medicine, WolaitaSodo University, P. O. Box 138, WolaitaSodo, Ethiopia \\ *Corresponding author: HabenFesseha, School of Veterinary Medicine, WolaitaSodo University, P. O. Box 138, Wolaita \\ Sodo, Ethiopia
}

ARTICLE INFO

Received: May 04, 2020

Published: 幽 June 02, 2020

Citation: Haben Fesseha. Mammary Mastectomy Due to Mammary Gland Tumors in Intact Female Dog. Biomed J Sci \& Tech Res 28(1)-2020. BJSTR. MS.ID.004589.

\begin{abstract}
Mammary tumors are the most common type of tumors in intact female dogs. Twelve years old female exotic breed dog was presented with a history of large-sized and hard swelling around the chest on left parts of the first mammary gland. Upon clinical examination, the case was tentatively diagnosed as a mammary gland tumor and a sample from the tumor mass was taken by excision for histopathological examination and confirmation. After proper restraining, aseptic procedure, and anesthetic protocol, the tumor mass surgically excised. Finally, the dog was successfully recovered and there was no report of recurrence during the follow-up. Surgical correction of mammary tumors in a female dog was described in this case report.
\end{abstract}

Keywords: Intact Female Dog; Histopathology; Mammary Tumor; Mastectomy

\section{Introduction}

Domestic animals were widely suffering from tumors of different origins. Mammary neoplasms are among the most common neoplasm in female dogs originating from the mammary gland. A tumor is an abnormal mass of tissue resulting from autonomous, progressive, excessive proliferation of body cells not integrated into normal tissue and exhibits varying degrees of fidelity to their precursors [1-3]. It may be classified by their primary site of origin, grade, stage (tumor size $(\mathrm{T})$, the degree of regional spread or node involvement $(\mathrm{N})$, distant metastasis (M) (TNM staging), and their histological or tissue types. However, the commonly used and most useful classification of tumors is histogenetic, that is, the tumors are named according to the tissues from which they arise and of which they consist [4].

Mammary tumors are most frequent in older intact bitches and queen, but they are found in other species of animals as well. There are two published articles (the first in 1974 and modification in 1999) that describe the histologic classification systems for canine mammary tumors and dysplasia in different species of animals (Goldschmidt et al., 2011). Mammary tumors were characterized by severe cellular and nuclear pleomorphism, and highly infiltrative that represents a serious problem in veterinary medicine $[3,5]$.
The exact cause of mammary tumors is poorly understood in any species including in dogs or cats; however, genetic and nutritional factors are considered as a possible cause. In mice, an oncornavirus is causative in certain inbred strains. In dogs, one single nucleotide polymorphism (SNP) in exon 9 of BRCA1 and one SNP in exon 24 of BRCA2 was found to be significantly associated with canine mammary tumors. The consumption of red meat and obesity are associated with an increased risk of mammary gland tumors in intact or ovariohysterectomized dogs $[1,6]$. Even though the exact mechanism is tumor genesis is unknown, estrogen or progesterone (or both) hormones play an important role in the hyperplasia and neoplasia of mammary tissue. They may also influence the pathogenesis of hormone-induced mammary neoplasia as well as the response to hormone therapy $[7,8]$.

The frequency of mammary neoplasia in different species varies tremendously. The dog is by far the most frequently affected domestic species, with a prevalence $\sim 3$ times that in women; $~ 50 \%$ of all tumors in the bitch are mammary tumors. Mammary tumors are rare in cows, mares, goats, ewes, and sows. There are differences in both biologic behavior and histology of mammary tumors in dogs and cats. Approximately $45 \%$ of mammary tumors are malignant in 
dogs, whereas $\sim 90 \%$ are malignant in cats, and dogs have a much higher number of complex and mixed tumors than do cats $[9,10]$.

The risk of developing mammary neoplasia can be reduced by ovariectomy before the first estrus. The risk can be minimized up to $0.5 \%$ in intact bitches whereas ovariectomy after one estrus reduces the risk to $8 \%$ of that in intact bitches. Bitches neutered after maturity have generally been considered to have the same risk as intact bitches. However, questions remain regarding the impact of ovariohysterectomy at the time of tumor excision and the timing of surgery relative to survival. In one study, dogs spayed less than 2 years old before tumor excision lived $45 \%$ longer than either intact dogs or those spayed greater than 2 years old before tumor excision $[11,12]$.

Canine mammary tumors (more than 50\%) are benign mixed tumors; however, a smaller percentage of malignant mixed tumors (epithelial or mesenchymal components or both) are seen. Histologically, canine mammary gland tumors have been classified by the World Health Organization as carcinomas (with six types and additional subtypes), sarcomas (four types), carcinosarcomas (mixed mammary tumors), or benign adenomas. This classification is based on the extent of the tumor, involvement of lymph nodes, and presence of metastatic lesions (TNM system); it includes unclassified tumors and benign dysplasias. In addition to tumor size and the status and timing of neutering, special stains (including those for the KIT receptor and AgNOR) may have prognostic value [13-15].

A mammary tumor is usually suspected of the detection of a mass during a physical examination and it commonly affects the two posterior mammary glands than the other three anterior glands. Grossly, tumors appear as single or multiple nodules (1$25 \mathrm{~cm}$ ) in one or more glands. The cut surface is usually lobulated, gray-tan, and firm, often with fluid-filled cysts. Mixed mammary tumors may contain grossly recognizable bone or cartilage on

\section{Case History and Presentation}
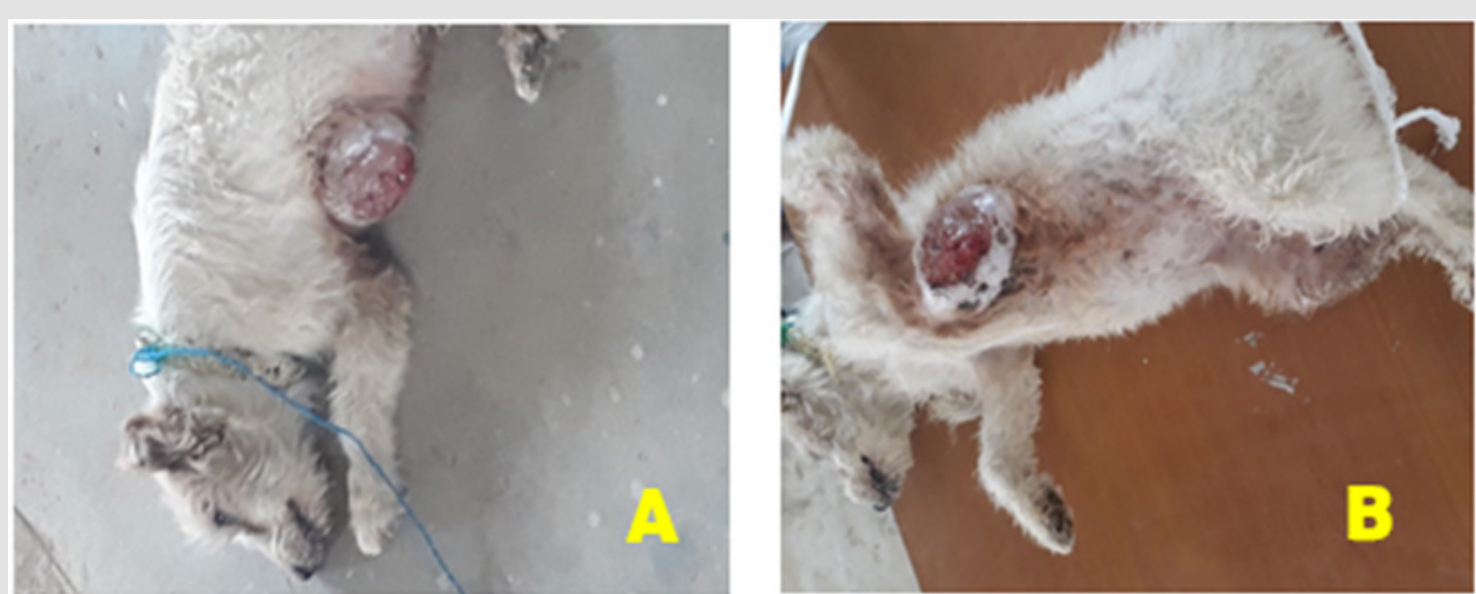

Figure 1:

A) A large-sized with hard mammary tumor mass on ventral thoracic area in dog.

B) Presentation of the tumor mass on thoracic pair of mammary glands after sedation. the cut surface [16]. The palpation of the regional lymph nodes can help determine the extent of spread. Thoracic radiographs, preferably three views (a ventral-dorsal and two laterals), should be taken to detect pulmonary metastases. Fine-needle aspirates may differentiate between inflammatory and neoplastic lesions but may lead to erroneous conclusions and delay of surgery. The diagnosis is determined by histopathology and is important in defining treatment and prognosis $[11,16,17]$.

Mammary tumors are treated surgically, although there is no consensus as to the best procedure. Removal of the tumor alone (lumpectomy), simple mastectomy (removal of the affected gland only), modified radical mastectomy (removal of the affected gland and those that share lymphatic drainage and associated lymph nodes), and radical mastectomy (removal of the entire mammary chain and associated lymph nodes) all have their proponents. In dogs, there have been case reports of measurable tumors responses to paclitaxel, doxorubicin, carboplatin, and mitoxantrone; although it is still uncertain whether adjuvant chemotherapy improves the survival times of dogs treated with mammary gland carcinomas post-operatively [18].

The prognosis is based on multiple factors such as type of tumors cell, stage of the tumors, size of the tumor, lymph node involvement, and nuclear differentiation. The length of time the mass has been present is usually unknown, but the rate of growth may help to determine the prognosis. Sarcomas are associated with shorter survival times than carcinomas. In cats, tumor size is important; cats with tumors $>3 \mathrm{~cm}$ in diameter have a median survival time of 6 months, but cats with tumors $<2 \mathrm{~cm}$ in diameter have a median survival time of greater than 4 years [2, 3]. A perusal of different kinds of literature, there are few published clinical studies and case reports in Ethiopia. This case report describes the surgical excision techniques of mammary gland tumors from the female dog. 
A twelve years old bitch weighing $12 \mathrm{~kg}$ was brought to the Mekelle University Veterinary Teaching Hospital with a history of swelling on the right anterior mammary glands on the lower part of the abdomen and the condition starts before 3 months. Moreover, the size of the swelling had grown progressively starting in the form of lumps with a lack of pain, limping, and licking of the site. Upon clinical presentations, a large-sized with hard mass swelling on her ventral thoracic area in between the left and right part of the first anterior the mammary glands (Figure $1 \mathrm{~A}$ ).

After a detailed clinical examination and thorough palpation, the tumor mass was located subcutaneously, painless, hard consistency, well-circumscribed and was attached to structures beneath it (Figure 1B). The clinical parameters like heart rate, respiration rate, and temperature were within physiological limits. The cut surface appeared multilobulated, papillary like projections, and white to grey in color. A biopsy sample of tumor mass was taken for histopathological examination of the lesions and the samples were preserved in 10\% neutral buffered formalin.

\section{Animal Handling, Preoperative Patient Prepara- tion, and Anesthetic Protocol}

Preoperatively, the dog was withheld $12 \mathrm{hrs}$ from feed and 6 hours from water before coming to the clinic based on an appointment. The dog then was properly restrained and premedicated with chemical methods using Ana-Sed® (xylazine $\mathrm{HCl}$, Heartland Vet Supply Ltd at dose rate of $1.1 \mathrm{mg} / \mathrm{kg}$, Intramuscularly). After proper sedation, the dog was positioned in lateral recumbency and the area around tumor mass was washed with water and soap thoroughly after deep sedation (Figure 2A). Then, the hair surrounding of the swelling was aseptically prepared by clipping, shaving, scrubbing, washing with water, and salvon $\AA$ (Cetrimide $3 \%$ and Chlorhexidine gluconate $0.5 \%$ solution).
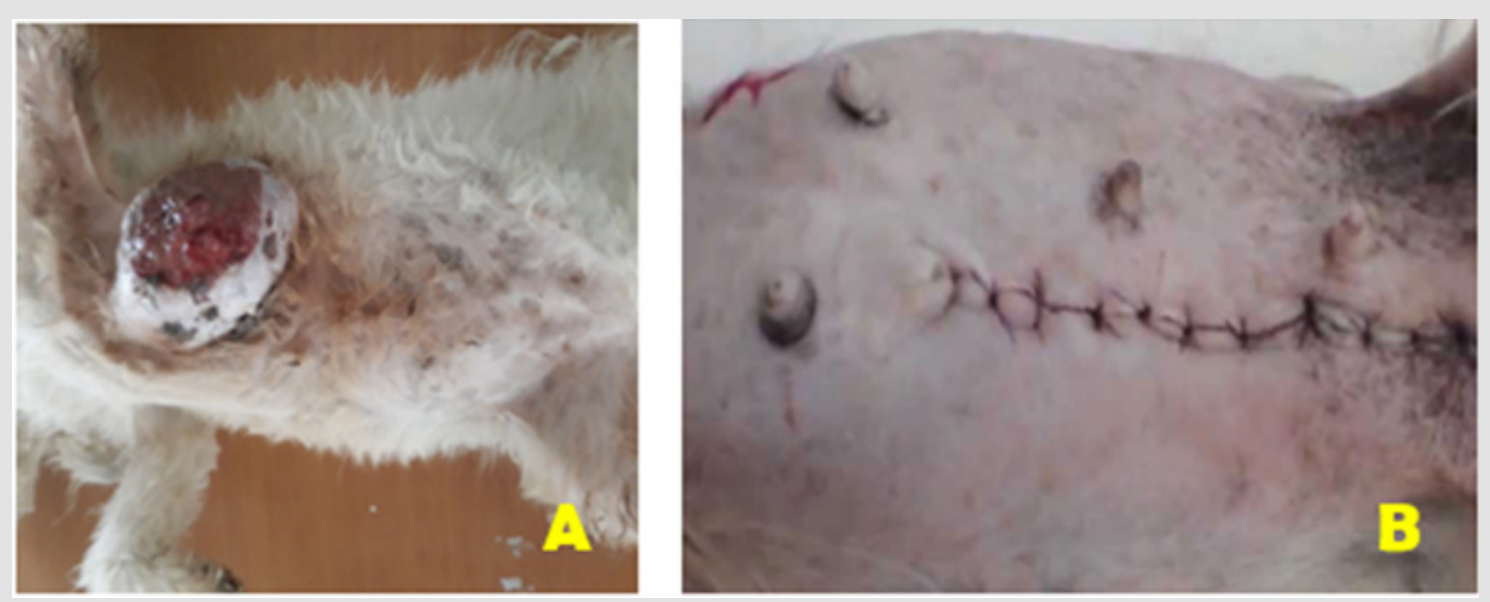

Figure 2:

A) Aseptic preparation of the surgical site with dorsolateral recumbency.

B) The post-surgical presentation of the surgical site in dog (Unilateral mastectomy).

The dog was kept on fluid therapy soon after induction (5\% Dextrose solution plus $0.9 \% \mathrm{NaCl}, 1000 \mathrm{ml}$ stat, I.V., Addis pharmaceutical, Adigrat Ethiopia, at a surgical rate of $10 \mathrm{ml} / \mathrm{kg} / \mathrm{hr}$. and calculated rate $1 \mathrm{drop} / \mathrm{sec}$ ) to maintain the acid base balance and minimize the risk of complication. Moreover, the dog was kept on antibiotic penicillin $(24 \mathrm{mg} / \mathrm{kg})$ and dihydrostreptomycin sulphate $\left(30 \mathrm{mg} / \mathrm{kg}\right.$ ) (PenStrep ${ }^{\circledR}$ Norbrook UK) I.M three hours preoperatively.

After proper premedication with Xylazine, the dog was induced by the cocktail of Xylazine (at dose rate of $1.1 \mathrm{mg} / \mathrm{kg}$ ) and Ketamine (Ketamine Hydrochloride, Germany) at $5 \mathrm{mg} / \mathrm{kg}$ I.M and maintained also by Ketamine and finally, the dogs were restrained in dorsolateral recumbency on a patient table where all legs were loosely secured for ease of surgical procedure. Finally, the surgical area was scrubbed three times with a povidone-iodine $1 \%$ solution to decrease the microbial load in the area and left dried till ready for excision of the tumor (Figure 1A).

\section{Laboratory Examination and Finding}

The mammary gland tumors in this case report was located on the caudal thoracic pairs of mammary glands. The gross characteristics of this tumor was well-circumscribed masses, diffuse swellings well attached to the body mass, and skin-covered to ulcerate in ventral side. Besides, the mass has had necrotic material with firm and irregular shape (Figure 2A).

Freshly collected canine mammary samples were sectioned transversely and examined grossly. Representative samples from gross lesions of testis were taken and fixed in $10 \%$ neutral buffered formalin (NBF) for $48 \mathrm{hrs}$. The samples were then trimmed and prepared in $1 \times 1 \mathrm{~cm}$ sizeand dehydrated in ascending concentrations of Ethyl alcohol, cleared in xylol followed by embedding in paraffin wax (melting point 55-580C). Paraffin blocks were prepared and serial sections from the middle part from each testis were taken at a thickness of $5 \mu \mathrm{m}$ using a rotatory microtome. These sections were stained with hematoxylin-eosin staining and were mounted 
on frosted-end glass slides using DPX mountant [19]. Finally, all the slides were microscopically examined under light microscope.

Histopathological examination of the biopsy of the tumors showed malignancy-free tissue, composed of higher nuclear to cytoplasm ratio, cellular pleomorphism, and prominent nucleoli (arrows in Figure 3) with increased numbers of mitotic figures (double nucleus and nuclear fragmentation). Finally, based on clinical and histopathogical findings, the case was diagnosed as a canine mammary tumor, and was decided to be undergone surgical excision of the mass (Unilateral mastectomy).

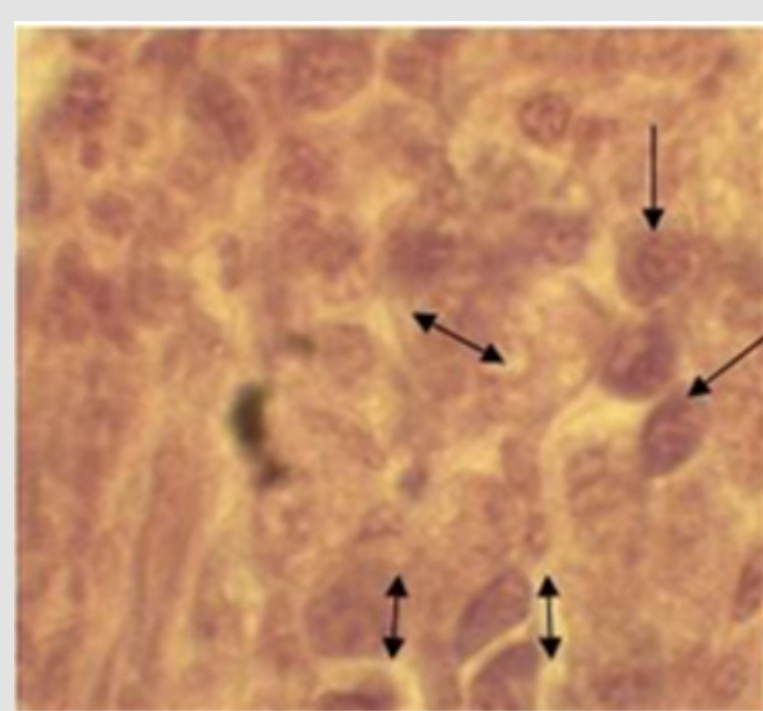

Figure 3: Histopathological appearance of mammary gland tumor.

\section{Surgical Correction}

After aseptically preparing each surgical site and keeping the dog in the appropriate position on the patient table, an elliptical skin incision was given on the base of the swelling and advanced gently with blunt dissection by using Metzenbaum scissor. After careful handling and divulsion of adjacent tissues, the skin, all tumorous mass, mammary tissue, and fat down to the rectus fascia was surgically excised. However, the rectus fascia or its underlying muscle was not removed. During dissection, bleeding during the procedure was managed by ligation of the caudal superficial epigastric artery and vein. In addition, applying sterile gauze, using different straight and curved hemostatic forceps and topical infiltration of epinephrine was applied bleeding. Then, the removed area was well cleaned before closure for both cases.

Before skin closure, obliteration of dead space was made through continuous apposing sutures of skin with subcutaneous tissue to prevent accumulation of serosanguinous fluid using polyglycolic acid 910 (vicryl) size 2/0. Finally, the skin was closed with ford interlocking suture pattern using polyglycolic acid 910 (vicryl) no. 2 (Figure 2B). Lastly, the area was properly cleaned and dressed in a $1 \%$ povidone-iodine solution and the dog was admitted home after full anesthetic recovery.

\section{Post-Operative Follow-up and Result}

Post-surgery, small skin bleeding was noticed but it is normal and helps to heal the wound unless continued for a long period. The owner was also advised to aseptically wash the surgical site at the second- and third-days post-operation. Besides, modified plastic collar was applied to avoid licking on the surgical site and Penicillin $(24 \mathrm{mg} / \mathrm{kg})$ and dihydrostreptomycin sulphate $(30 \mathrm{mg} /$ $\mathrm{kg}$ ) (PenStrep ${ }^{\circledR N}$ Norbrook UK) I.M was administered for three consecutive three days. Meloxicam, at a dose rate of $0.5 \mathrm{mg} / \mathrm{kg}$, IM) was given twice daily for three successive days for pain management. Moreover, the owners were also advised to subjectively assess for discomfort, unpleasant discharges, pain, and inflammation at the surgical site. No signs of recurrence were present during this period. The postoperative evaluations showed good healing progress of the surgical wound and good general condition of the patients, as well.

\section{Discussion and Conclusion}

Mammary tumors are common in dogs and cats. Surgical techniques for mammary tumor removal include lumpectomy, mammectomy, regional mastectomy, unilateral mastectomy, bilateral mastectomy, and radical mastectomy. The ideal technique for each case depends on the species and the number, size, and location of mammary tumors. In all procedures, the surgical wound should be closed in 2 to 3 layers (deep SC tissue, SC tissue, skin) [8, $10,20]$. This was in line with the current case report in terms of case handling and management.

Mammary neoplasms are the most common neoplasms in female dogs (Goldschmidt et al.,2011) comprising up to $25-30 \%$ of all neoplasms in dogs [21], and mixed tumors and carcinoma are diagnosed in $50-65 \%$ and $25-40 \%$ of cases of mammary tumors, respectively $[9,21]$. Moreover, the majority of the mammary gland tumors macroscopically showed a hard or an elastic consistency but some of them appeared fluctuant with edema or necrotic areas. Moreover, in the great majority of cases, the aspect of the tumor on the cut surface was white to grey and lobed [22]. This report was in agreement with this study.

Histologically, the mammary gland tumors showed malignancyfree tissue, composed of higher nuclear to cytoplasm ratio, cellular pleomorphism, and prominent nucleoli with increased numbers of mitotic figures. This general feature of tumors in this study agreed to the report by Goldschmidt et al. [17] who described similar histological characteristics including hypercellularity, hyperplasia, hyperchromasia, nuclear and cellular pleomorphism, high nuclear to cytoplasmic ratio and moderate to severe mitotic figures with prominent nucleoli in canine.

Unilateral mastectomy was performed that involves removal of all mammary tissue on one side of midline (Figure 2B). This includes lumpectomy (remove all skin), mammary tissue, and fat down to the rectus fascia without removing the rectus fascia. Moreover, there was no recurrence of tumor. The reason for non-recurrence 
in the present case might be due to its complete excision of the tumor mass as it was reported by different authors [6,8,23]. Canine mammary gland tumors are common tumors in dog that need an accurate diagnosis, complete surgical excision (mastectomy) of the tumor mass and proper follow-up of the case are essential to prevent recurrence and restore function.

\section{Conflict of Interest}

Author has no disclosure to make that qualifies as a conflict of interest.

\section{References}

1. RubinR, et al. (2008) Rubin's pathology: clinicopathologic foundations of medicine. Lippincott Williams \& Wilkins, USA.

2. Dantas CassaliG, Angélica Cavalheiro Bertagnolli, Enio Ferreira,Karine Araújo Damasceno, Conrado de Oliveira Gamba, et al. (2012) Canine mammary mixed tumours: A review. Veterinary medicine international: $1-7$.

3. GardenO, SW Volk, NJ Mason 3, JA Perry (2018) Companion animals in comparative oncology: One Medicine in action. The Veterinary Journal 240: 6-13.

4. JoshiS, et al. (2012) Introduction to neoplasm: 'tumor classification'a review article. International Journal of Advanced Research in Pharmaceutical \& Bio Sciences 1: 227-264.

5. Information for Oncology Clients (2018) Mammary Tumors in Dogs Clinical Oncology Service Ryan Veterinary Hospital of the University of Pennsylvania.

6. Kamble M, et al. (2016) Surgical management of mammary tumor in a dog. Intas Polivet 17: 196-197.

7. McEnteeMC (2002) Reproductive oncology. Clinical techniques in small animal practice 17: 133-149.

8. PapazoglouL, Eleni Basdani, Stefania Rabidi, Michael N Patsikas, Maria Karayiannopoulou (2014) Current surgical options for mammary tumor removal in dogs. J Vet Sci Med 2: 2-7.

9. AlenzaMP, L Peña, N del Castillo, A I Nieto (2000) Factors influencing the incidence and prognosis of canine mammary tumours. Journal of Small Animal Practice 41: 287-291.

10. MorrisonWB (2002) Cancer in dogs and cats: medical and surgical management. Teton New Media.

ISSN: 2574-1241

DOI: $10.26717 /$ BJSTR.2020.28.004589

Haben Fesseha. Biomed J Sci \& Tech Res

This work is licensed under Creative Commons Attribution 4.0 License

Submission Link: https://biomedres.us/submit-manuscript.php
11. McCarthy A, et al. (2003) Canine mammary carcinoma. Online resources of the Department of Pathology, University of Georgia College of Veterinary Medicine.

12. StratmannN, Klaus Failing, Andreas Richter, Axel Wehrend (2008) Mammary tumor recurrence in bitches after regional mastectomy. Veterinary Surgery 37: 82-86.

13. KimuraKC, APGárate, Maria Dagli (2012) Retrospective study of neoplasms in domestic animals: A survey between 1993 and 2002 of the service of animal pathology, department of pathology, school of veterinary medicine and animal science, University of Sao Paulo, Southeast Brazil. Brazilian Journal of Veterinary Pathology 5: 60-69.

14. Sorenmo K (2003) Canine mammary gland tumors. Veterinary Clinics: Small Animal Practice 33: 573-596.

15. MarconatoL, Giorgio Romanelli, Damiano Stefanello, Claudio Giacoboni, Ugo Bonfanti, et al. (2009) Prognostic factors for dogs with mammary inflammatory carcinoma: 43 cases (2003-2008). Journal of the American Veterinary Medical Association 235: 967-972.

16. ShafieeR, Javad Javanbakht, Nahid Atyabi , Pegah Kheradmand, Danial Kheradmand ,et al. (2013) Diagnosis, classification and grading of canine mammary tumours as a model to study human breast cancer: An Clinico-Cytohistopathological study with environmental factors influencing public health and medicine. Cancer cell international 13: 79.

17. Goldschmidt M, L Peña, R Rasotto, V Zappulli (2011) Classification and grading of canine mammary tumors. Veterinary pathology 48: 117-131.

18. CassaliGD, Lidianne Narducci Monteiro, Conrado de Oliveira Gamba, Karine Araújo Damasceno, Cecília Bonolo de Campos, et al. (2015) Cytologic analysis of the mammary papillar discharge in a canine micropapillary carcinoma. Veterinary clinical pathology 44: 448-451.

19. Talukder S (2007) Histopathology techniques: Tissue processing and staining. Mymensingh Medical College, Department of Pathology, Mymensingh: 11.

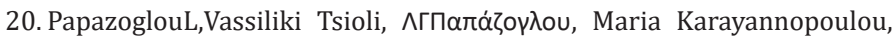
Ioannis Savvas, et al. (2006) Comparison of three methods for closure of mastectomy incisions in dogs. Aust. Vet. J 36: 156-162.

21. HazirogluR,B. Yardimci, S Aslan, MZ Yildirim, N Yumusak, et al. (2010) Cytological Evaluation of canine mammary tumours with fine needle aspiration biopsy. Revue Méd. Vét 161: 212-218.

22. Allen SW, K W Prasse, E A Mahaffey (1986) Cytologic differentiation of benign from malignant canine mammary tumors. Veterinary pathology 23: 649-655.

23. RaoCM, B chandra prasad, NVV Hari Krishna (2011) Surgical Management of Lipoma in a Dog. Veterinary World 4: 34.

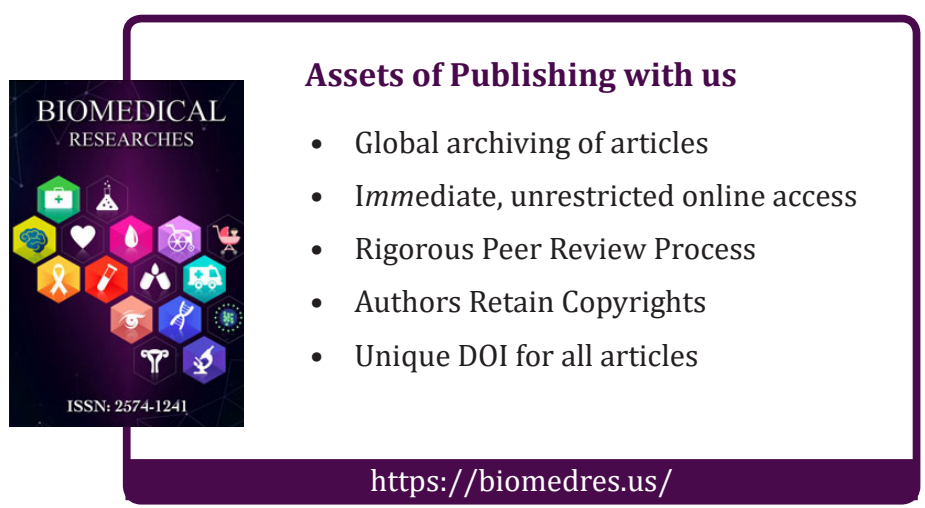

